\title{
From confined spinons to emergent fermions: Observation of elementary magnetic excitations in a transverse-field Ising chain
}

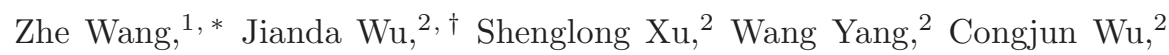 \\ Anup Kumar Bera, ${ }^{3, \text { f }}$ A. T. M. Nazmul Islam, ${ }^{3}$ Bella Lake, ${ }^{3,4}$ Dmytro Kamenskyi, ${ }^{5}$ \\ Papori Gogoi, ${ }^{5}$ Hans Engelkamp, ${ }^{5}$ Nanlin Wang, ${ }^{6,7}$ Joachim Deisenhofer, ${ }^{1}$ and Alois Loidl ${ }^{1}$ \\ ${ }^{1}$ Experimental Physics $V$, Center for Electronic Correlations and Magnetism, \\ Institute of Physics, University of Augsburg, 86135 Augsburg, Germany \\ ${ }^{2}$ Department of Physics, University of California, San Diego, California 92093, USA \\ ${ }^{3}$ Helmholtz-Zentrum Berlin für Materialien und Energie, 14109 Berlin, Germany \\ ${ }^{4}$ Institut für Festkörperphysik, Technische Universität Berlin, 10623 Berlin, Germany \\ ${ }^{5}$ High Field Magnet Laboratory (HFML-EMFL), Radboud University, 6525 ED Nijmegen, The Netherlands \\ ${ }^{6}$ International Center for Quantum Materials, School of Physics, Peking University, 100871 Beijing, China \\ ${ }^{7}$ Collaborative Innovation Center of Quantum Matter, Beijing, China
}

(Dated: September 20, 2016)

\begin{abstract}
We report on spectroscopy study of elementary magnetic excitations in an Ising-like antiferromagnetic chain compound $\mathrm{SrCo}_{2} \mathrm{~V}_{2} \mathrm{O}_{8}$ as a function of temperature and applied transverse magnetic field up to $25 \mathrm{~T}$. An optical as well as an acoustic branch of confined spinons, the elementary excitations at zero field, are identified in the antiferromagnetic phase below the Néel temperature of $5 \mathrm{~K}$ and described by a one-dimensional Schrödinger equation. The confinement can be suppressed by an applied transverse field and a quantum disordered phase is induced at $7 \mathrm{~T}$. In this disordered paramagnetic phase, we observe three emergent fermionic excitations with different transverse-field dependencies. The nature of these modes is clarified by studying spin dynamic structure factor of a 1D transverse-field Heisenberg-Ising (XXZ) model using the method of infinite time evolving block decimation. Our work reveals emergent quantum phenomena and provides a concrete system for testifying theoretical predications of one-dimension quantum spin models.
\end{abstract}

\section{INTRODUCTION}

Emergent states of matter in quantum magnets are characterized by their elementary excitations that can be induced and tuned in an external magnetic field. Due to enhanced quantum fluctuations and reduced dimensionality, the low-dimensional quantum magnets host very often exotic quantum states of matter ${ }^{1-13}$. Especially for the one-dimensional (1D) spin systems, realization of novel quantum spin states is not only of lively experimental interest ${ }^{7-10,12,13}$ but also a constant focus of theoretical study ${ }^{1,2,14-17}$, since quantitative description of the elementary excitations can be provided by rigorous theoretical approaches and precise comparisons between theoretical predictions and experimental results can be made $-10,12,13,15$.

In the $1 \mathrm{D}$ spin- $1 / 2$ systems, the elementary magnetic excitations are spinons with fractional spin quantum number $S=1 / 2^{18}$. Confinement of the fractional spinon excitations can be even realized $9,10,19-22$ as an analogy with quark confinement in particle physics 23 . The concept of spinon confinement is illustrated in [Fig. 1](a)(d)]. In a classical picture, the ground state of a spin$1 / 2$ Ising antiferromagnetic chain corresponds to an antiparallel alignment of neighboring spins [Fig. 11(a)]. A single spin flip frustrates the intra-chain exchange interactions $J$ and creates two spinons, each with spin1/2 [Fig. 1(b)]. Subsequent spin flips can lead to propagation of the spinons along the chain [Fig. 1(c)]. In the presence of weak inter-chain exchange interactions $J_{\perp} \ll J$ [Fig. 1(d)], a 3D Néel antiferromagnetic order can be stabilized at low temperature $T<T_{N}$ and the separation of spinons from each other is unfavorable due to the frustrated inter-chain couplings. Thus the two spinons feel a confining potential, increasing with the distance between them, and form quantized spinon bound states $[\text { Fig. } 1 \text { (e)(f) }]^{24,25}$. Since the spinons are confined by the inter-chain couplings only in the antiferromagnetic phase, the magnetic phase transition can be thought as accompanied by a spinon confinement-deconfinement transition.

$\mathrm{SrCo}_{2} \mathrm{~V}_{2} \mathrm{O}_{8}$ is a representative realization of the paradigmatic 1D Heisenberg-Ising (XXZ) model. It crystallizes in the tetragonal structure with space group $\mathrm{I}_{1}$ cd. Screw spin chains in $\mathrm{SrCO}_{2} \mathrm{~V}_{2} \mathrm{O}_{8}$ are constituted by edge-sharing $\mathrm{CoO}_{6}$ octahedra and propagate along the crystallographic $c$ axis [Fig. [1(g)] 26,27. Each screw period consists of four $\mathrm{Co}^{2+}$ ions with effective spin $S=1 / 2$, corresponding to the lattice constant $c$. The unit cell contains four screw chains with either left- or right-hand chirality [Fig. 1(h)]. Due to spin-orbit coupling and crystal field splitting, the $\mathrm{Co}^{2+}$ spins have an Ising-like anisotropy along the $c$ axis $^{26,27}$. The dominant exchange interaction $J$ is antiferromagnetic and between the nearest-neighbor $\mathrm{Co}^{2+}$ spins in the chain. The interchain coupling $J_{\perp}$ is much smaller and dominated by exchange between the nearest-neighbor $\mathrm{Co}^{2+}$ ions in the $a b$ plane that are from the neighboring chains with the same chirality [Fig. [1(h)]. At $T_{N}=5 \mathrm{~K}$ a long-range Néel-type antiferromagnetic order along the chain is stabilized [Fig. [1(g) $]^{26-29}$.

Below $T_{N}$ confinement of spinons is characterized by 
a series of quantized spinon bound states [Fig. 1(f) $]^{22}$, which with spin $S=+1$ or -1 can be split in longitudinal magnetic field and exhibit linear field dependence 20,22 . By applying a transverse magnetic field, the Néel temperature can be reduced according to the magnetic susceptibility measurements $26,27,30-32$. At $2 \mathrm{~K}$, a phase transition from the Néel antiferromagnetic (AFM) phase to a disordered paramagnetic (PM) phase is induced by a transverse field of $7 \mathrm{~T}^{26,27}$.

The reduction of Néel temperature suggests that the applied transverse magnetic field competes with the weak inter-chain couplings, and thus reduces the confining effects. This motivates us to utilize a transverse field to tune the spinon confinement. By continuously changing the transverse field, the confinement is expected to be suppressed with deconfined spinons observed.

Here we perform terahertz spectroscopy on the low-energy spin excitations in the $1 \mathrm{D}$ Ising-like antiferromagnetic-chain $\mathrm{SrCo}_{2} \mathrm{~V}_{2} \mathrm{O}_{8}$ as a function of transverse magnetic field, supplemented by studying spin dynamics of the corresponding 1D transverse-field XXZ model using the rigorous method of infinite time evolving block decimation (iTEBD). In zero field and below $T_{N}$, confinement of spinons of optical branch as well as of acoustic branch is observed and identified. Both the optical and the acoustic series of confined spinons can be described by a 1D Schrödinger equation with linear confining potential. In small transverse fields, where the system remains antiferromagnetically ordered, the confining effects are found to be reduced: The confining potential becomes shallower with increasing field and the higher energy spinon bound states tend to collapse on the lowest level. At the transverse-field-induced orderdisorder transition, we observe collapse of the quantized confined-spinon levels. Above this transition, the lowenergy spin excitations in $\mathrm{SrCo}_{2} \mathrm{~V}_{2} \mathrm{O}_{8}$ are not deconfined spinons, but characterized by emergent fermions of the 1D transverse-field Ising class. We successfully identify the observed emergent fermions and their field dependencies by performing theoretical study of the $1 \mathrm{D}$ spin- $1 / 2$ XXZ model using the iTEBD method.

\section{EXPERIMENTAL DETAILS AND THEORETICAL METHOD}

High-quality single crystals of $\mathrm{SrCo}_{2} \mathrm{~V}_{2} \mathrm{O}_{8}$ were grown using the floating-zone method and charactized by Xray diffraction, neutron diffraction, and magnetization measurements 26 . Single crystals for the optical study were oriented using Laue diffraction and cut perpendicular to the tetragonal $a$ axis with a typical surface of $4 \times 4 \mathrm{~mm}^{2}$ and a thickness of $1 \mathrm{~mm}$. High-field optical measurements were performed in the High Field Magnet Laboratory in Nijmegen. A Michelson interferometer with mercury lamp was used to generate electromagnetic waves in the terahertz frequency range which were detected by a silicon bolometer cooled at $1.6 \mathrm{~K}$. The sam-
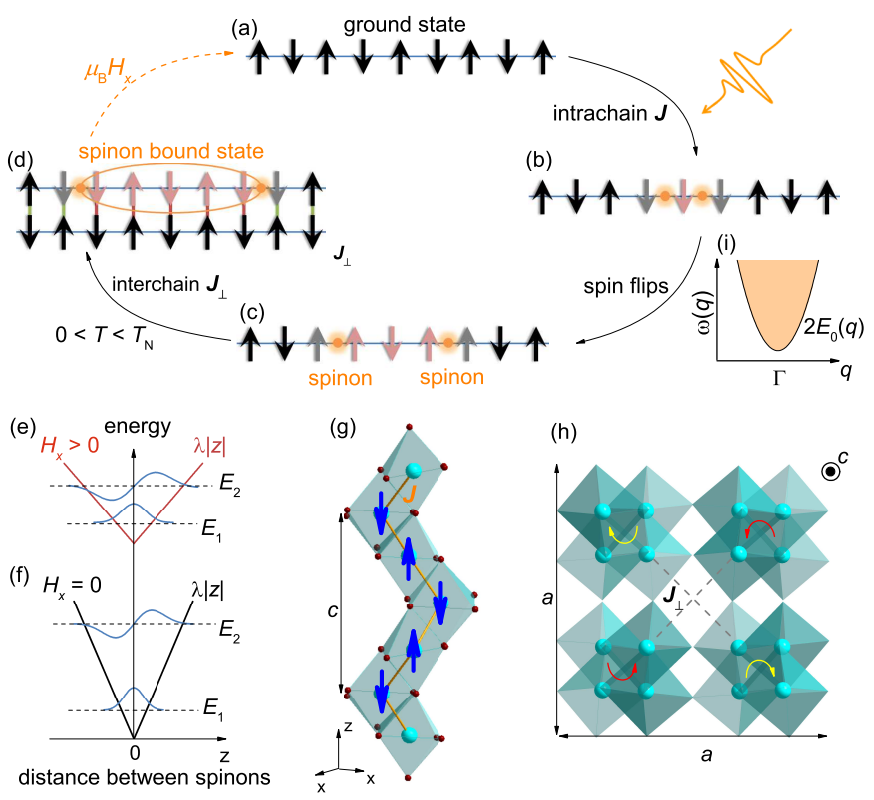

Figure 1. (a) Ground state of an antiferromagnetic Ising chain with antiparallel alignment of neighboring spins. (b) A single spin-flip excitation composed of two spinons (orange dots). (c) Spinons can propagate along the chain by subsequent spin flips due to $x y$ components of the exchange interactions. (d) Two spinons form a spinon bound state due to the frustration of inter-chain exchange $J_{\perp}$. (e) and (f) Potential energy increases linearly with distance between spinons $V(z)=\lambda|z|$ with $\lambda=2 J_{\perp}\left\langle S_{z}\right\rangle^{2} / c$ and $c$ is the lattice constant along the chain direction, covering four spin sites in $\mathrm{SrCo}_{2} \mathrm{~V}_{2} \mathrm{O}_{8}$ [see (g) and (h)]. The two lowest-lying spinon bound states with energies $E_{1}$ and $E_{2}$ are schematically shown for zero and finite transverse fields $H_{x}$ with $\lambda\left(H_{x}=0\right)>\lambda\left(H_{x}>0\right)$. (g) Screw chain of edge-shared $\mathrm{CoO}_{6}$ octahedra propagating along the crystallographic $c$ axis in $\mathrm{SrCo}_{2} \mathrm{~V}_{2} \mathrm{O}_{8}$ with antiferromagnetic order of $\mathrm{Co}^{2+}$ spins along the $c$ direction stabilized below the Néel temperature $T<T_{N}$. (h) Viewing of left- and righthanded screw chains along the $c$ direction. The dominant interchain exchange $J_{\perp}$ is between the nearest-neighbor Co ions in the $a b$ plane, which are from the chains with the same chirality. $\quad J_{\perp}>0$ is antiferromagnetic. (i) Parabolic dispersion relation of the energy threshold $2 E_{0}$ in the reciprocal space close to the $\Gamma$ point $(q=0)^{24,25}$.

ple transmission spectrum was measured in the spectral range of $1.2-10 \mathrm{meV}(0.3-2.5 \mathrm{THz})$ at magnetic fields up to $25 \mathrm{~T}$. Magnetic fields were applied perpendicular to the crystallographic ac plane and parallel to the propagation direction of the electromagnetic wave (Faraday configuration).

Standard infinite time evolving block decimation (iTEBD) method 33.34 was used to study spin dynamics of the 1D XXZ antiferromagnetic model in a transverse field. This method has been applied to other 1D systems ${ }^{33.35}$ and turned out to be efficient to study 1D spin dynamics. Similar to the method of density matrix renormalization group, the iTEBD method assumes 

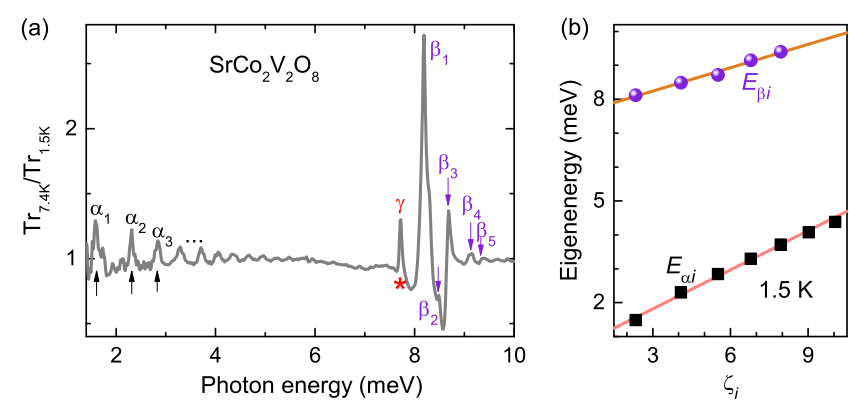

Figure 2. (a) Ratio of transmission spectra that are measured at $7.4 \mathrm{~K}$ (above $T_{N}$ ) and at $1.5 \mathrm{~K}$ (below $T_{N}$ ). Two series of excitations $\alpha_{i}$ and $\beta_{i}$ are observed and indicated by the arrows, corresponding to confined spinons of acoustic and optical branch, respectively. Another mode $\gamma$ at $7.72 \mathrm{meV}$ is marked by the star. (b) Eigenenergies $E_{\alpha i}$ and $E_{\beta i}$ of the modes $\alpha_{i}$ and $\beta_{i}$, respectively, as a function of $\zeta_{i}$ the negative zeros of the Airy function ${ }^{36}$. Linear fits according to the 1D Schrödinger equation are shown by the solid lines [see Eqs. (3) and (4)].

that the ground state (GS) of the spin system can be expressed as a matrix product state ${ }^{33,34}$. The two-point correlation function

$$
C(r, t)=\left\langle S_{y}(r, t) S_{y}(0,0)+S_{z}(r, t) S_{z}(0,0)\right\rangle_{G S}
$$

is calculated to compare with experimental results. The spin components $S_{y}$ and $S_{z}$ are coupled to the THz magnetic field and the applied external magnetic field is set along the $x$ direction. The spin dynamic structure factor (DSF) can be obtained via Fourier transformation

$$
S^{y y+z z}(q, \omega)=\int_{-\infty}^{\infty} d r \int_{-\infty}^{\infty} d t C(r, t) e^{i(\omega t-q r)}
$$

corresponding to the magnetic-dipole excitations observed in the optical measurements. In our calculation, the bond dimension is taken to be 40 and the discrete time step in the real time evolution is $0.02 / J$. The maximal time difference $\left|t-t^{\prime}\right|$ is set to $32 / J$, and we apply the linear prediction method ${ }^{35}$ to further improve the resolution.

\section{EXPERIMENTAL RESULTS}

\section{A. Confinement of acoustic and optical spinons in zero field}

A spin-flip excitation can be triggered by absorbing a photon, when the photon has the same energy as the two spinons created [Fig. 1(a)(b)]. The resonance absorption of photons is manifested by a transmission minimum (or an absorption maximum) at the corresponding photon energy. After the creation, the two spinons can propagate along the chain [Fig. 1(c)]. In presence of an effective linear confining potential $\lambda|z|$, the two-spinon bound state $\varphi(z)$ as a function of the distance $z$ between them can be described by the one-dimensional Schrödinger equation

$$
-\frac{\hbar^{2}}{\mu} \frac{d^{2} \varphi}{d z^{2}}+\lambda|z| \varphi=\left(E-2 E_{0}\right) \varphi
$$

with $\hbar$ the Planck constant, $\mu$ an effective mass, and $2 E_{0}$ the energy threshold for creating two spinons. $\lambda=$ $2 J_{\perp}\left\langle S_{z}\right\rangle^{2} / c$ is an effective description of the inter-chain exchange interaction $J_{\perp}$, with the lattice constant $c$. The solution of Eq. (3) is given by the linear dependence of $E_{i}$ on $\zeta_{i}$

$$
E_{i}=2 E_{0}+\zeta_{i} \lambda^{2 / 3}\left(\frac{\hbar^{2}}{\mu}\right)^{1 / 3}
$$

where $i=1,2,3, \ldots$ and $\zeta_{i}$ are given by the negative zeros of the Airy function $A i\left(-\zeta_{i}\right)=0 \underline{36}$.

Figure 2(a) shows the ratio of two transmission spectra of $\mathrm{SrCo}_{2} \mathrm{~V}_{2} \mathrm{O}_{8}$ that are measured above and below the Néel temperature $T_{N}=5 \mathrm{~K}$. Spin excitations due to the magnetic phase transition are manifested by the peaks. One can see three groups of excitations that are denoted by $\alpha_{i}, \beta_{i}$, and $\gamma$. Eigenenergies $E_{\alpha i}$ of the $\alpha_{i}$ series follow the linear dependence on $\zeta_{i}$ as given in Eq. (4) with $2 E_{0}=0.68 \mathrm{meV}$ and $\lambda^{2 / 3}\left(\frac{\hbar^{2}}{\mu}\right)^{1 / 3}=0.38 \mathrm{meV}$ as shown in Fig. 2(b). This indicates that the $\alpha_{i}$ series is confined spinon excitations, which is consistent with previous study $\underline{22}$. In the same representation [Fig. 2(b)], one can see that the $\beta_{i}$ modes with higher eigenenergies $E_{\beta i}$ also follow a linear dependence and the linear fit is given by $2 E_{0}=7.55 \mathrm{meV}$ and $\lambda^{2 / 3}\left(\frac{\hbar^{2}}{\mu}\right)^{1 / 3}=0.23 \mathrm{meV}$. Thus, the $\beta_{i}$ modes can be identified as another branch of confined spinons. Compared to the $\alpha_{i}$ series, the $\beta_{i}$ modes have higher energy threshold and larger effective mass, if the same confining potential is assumed for both branches.

It will be shown in the following that we are able to assign the $\alpha_{i}$ modes as confined spinons of acoustic branch and the $\beta_{i}$ modes as of optical branch, in analogy with the concept of optical and acoustic phonons. In contrast, the mode $\gamma$ at $7.72 \mathrm{meV}$ does not exhibit a series of excitations with higher energies and thus not fit into the same scheme as the $\alpha_{i}$ and $\beta_{i}$ modes. It is worth noting that the $\beta_{i}$ modes are observed in the same energy range as the lower-lying optical phonon bands, making the higher energy levels difficult to resolve.

\section{B. Tuning the spinon confinement in low transverse magnetic field}

Figures 3(a) and 3(b) show transmission spectra of the acoustic $\alpha_{i}$ and optical $\beta_{i}$ spinons of $\mathrm{SrCo}_{2} \mathrm{~V}_{2} \mathrm{O}_{8}$ as a function of applied transverse magnetic field along 


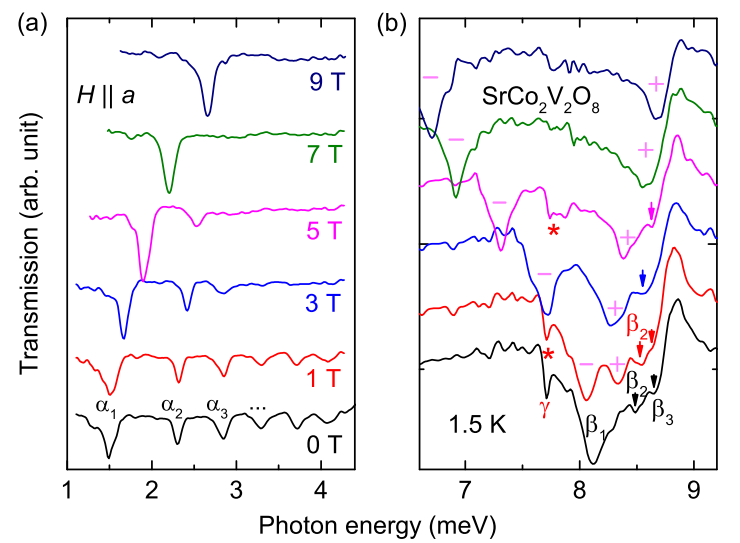

Figure 3. Transmission spectra of (a) the acoustic $\alpha_{i}$ and (b) the optical $\beta_{i}$ spinons in applied transverse magnetic fields along the crystallographic $a$ axis $(H \| a)$ up to 9 T. Series of absorption modes $\alpha_{i}$ can be observed at each field in (a). In (b) the zero-field mode $\beta_{1}$ splits into two modes in magnetic fields due to Zeeman interaction, as marked by the plus and minus signs. Below $7 \mathrm{~T}$, shifting of the mode $\beta_{2}$ to higher energy with increasing fields is observed and indicated by the arrows. The $\gamma$ mode at $7.72 \mathrm{meV}$, marked by the star, has almost field-independent eigenenergy below $7 \mathrm{~T}$ and is not resolvable at $7 \mathrm{~T}$ or above.

the crystallographic $a$ axis $(H \| a)$ up to $9 \mathrm{~T}$. At zero field the series of transmission minima, corresponding to confined acoustic and optical spinons, can be identified in agreement with the temperature-dependent measurements [Fig. 2(a)]. For the acoustic branch [Fig. 3(a)], a series of transmission minima for $1 \mathrm{~T}$ is observed at almost the same energies as for $0 \mathrm{~T}$, while at $3 \mathrm{~T}$ the resolved modes are shifted to higher energies [see also Fig. 4(e)]. Interestingly, the dependence of the resonance energies $E_{i}$ on $\zeta_{i}$ still follows the characteristic dependence at finite transverse fields, as shown in Figs. 4(a) and 4(b). This indicates that the spinon confinement is still a valid description of the spin dynamics in low transverse fields. The linear fit determines the parameters $\lambda^{2 / 3}\left(\frac{\hbar^{2}}{\mu}\right)^{1 / 3}=0.36 \mathrm{meV}$ and $2 E_{0}=0.87 \mathrm{meV}$ for $3 \mathrm{~T}$ [Fig. 四(b)], where the former decreased while the latter significantly increased compared to the results at zero field. Thus, by applying a transverse magnetic field, one not only changes the energy threshold of the confined spinons, but also tunes the profile of the confining potential.

As shown in Figs. 4(c) and 4(d), the confining potential and threshold energy show a non-linear decrease and increase, respectively, with increasing transverse field. This is different from the situation of small longitudinal fields along the Ising axis, where the threshold energy exhibits Zeeman-type linear dependence on field and the confining-potential profile is almost unchanged ${ }^{20,22}$, since the $U(1)$ symmetry of the spin Hamiltonian is broken in the transverse field. The results in Figs. 4(c) and 4(d) suggest that the confining potential becomes shallower in the transverse field. In a shallower confining potential, as illustrated in Figs. 1(e)(f), the difference between two neighboring spinon bound states reduces. When the difference becomes very small, the higher level cannot be distinguished experimentally. With increasing transverse field, the highest resolvable mode shifts to lower energy levels and, finally, when the second level $\left(\alpha_{2}\right)$ collapses onto the first one $\left(\alpha_{1}\right)$, the spinon hierarchy breaks down and the spinon confinement is completely suppressed.

As shown in Fig. 3(b), the zero-field optical mode $\beta_{1}$ splits into two in the transverse fields due to Zeeman interaction, one shifting up while the other shifting down, as marked by the plus and minus signs. The higherlevels of confined optical spinons should be also split in the transverse field, since they belong to the same series. Due to strong phonon absorption at the same spectral range, fewer up-shifting confined modes of the optical branch can be resolved by the field-dependent measurements [marked by the arrows in Fig. 3(b)], while higher levels of the down-shifting modes are covered by the strong $\beta_{1}$ absorptions. Nevertheless, one can clearly observe some similar tuning behavior of the confined optical spinons as of the acoustic modes: The higher-level modes move towards the first level with increasing transverse field, although they all shift to higher energies with increasing fields; Above $3 \mathrm{~T}$ the $\beta_{3}$ mode cannot be distinguished from the $\beta_{2}$ mode, and at $7 \mathrm{~T}$ only the main modes $\left(\beta_{+}\right.$and $\left.\beta_{-}\right)$can be observed indicating the sup-
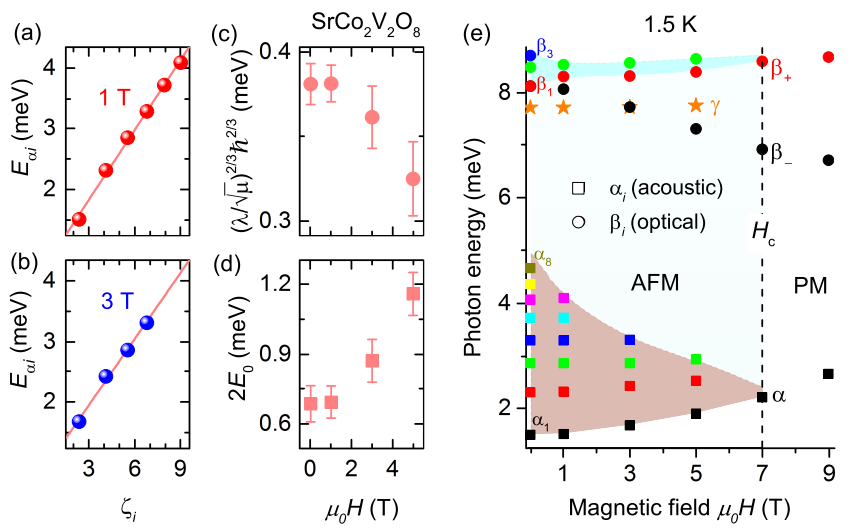

Figure 4. (a) and (b) Eigenenergies $E_{\alpha i}$ of the absorption modes $\alpha_{i}$ as a function of $\zeta_{i}$ for $1 \mathrm{~T}$ and for $3 \mathrm{~T}$. The lines are linear fits according to the 1D Schrödinger equation with linear confining potential [Eqs. (3) and (4)]. (c) and (d) The fitting parameters $\lambda^{2 / 3}\left(\frac{\hbar^{2}}{\mu}\right)^{1 / 3}$ and $2 E_{0}$ as functions of the transverse magnetic field. (e) Eigenenergies of the acoustic $\alpha_{i}$, the optical $\beta_{i}$ spinons and the $\gamma$ mode as functions of the magnetic field. At $7 \mathrm{~T}$, the higher levels collapse to the lowestlevel mode, $\alpha$ for the acoustic and $\beta_{+}$for the optical spinons; the $\gamma$ mode is suppressed; and only three modes $\alpha, \beta_{+}$, and $\beta_{-}$ remain. This indicates a field-induced phase transition from the antiferromagnetically (AFM) ordered to a paramagnetic (PM) quantum-disordered phase $\frac{26,27}{2}$ at $H_{c}=7 \mathrm{~T}$. 

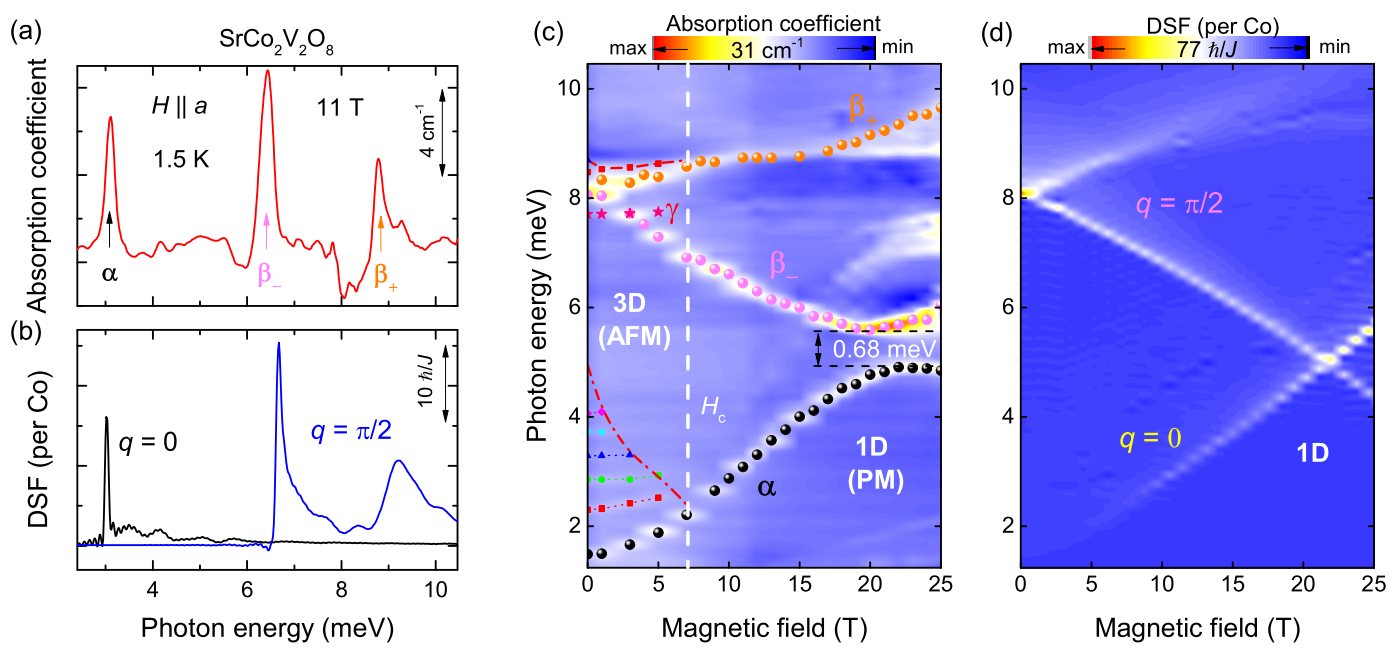

Figure 5. (a) Absorption-coefficient spectrum measured in transverse magnetic field of $11 \mathrm{~T}$ at $1.5 \mathrm{~K}$, exhibiting three spin excitation modes $\alpha, \beta_{-}$, and $\beta_{+}$. The peak positions of the three modes are shown in (c) as a function of magnetic field (dots). (b) Spin dynamic structure factor (DSF) at $q=0$ and $q=\pi / 2$ as a function of energy in the corresponding transverse magnetic field, simulated by the iTEBD method ${ }^{33,34}$ based on the $1 \mathrm{D}$ spin-1/2 XXZ model in Eq. (5). (c) Absorption coefficient and (d) DSF at $q=0$ and $q=\pi / 2$ as a function of photon energy and transverse magnetic field. The vertical dashed line at $H_{c}=7 \mathrm{~T}$ indicates the field-induced phase transition from the 3D antiferromagnetic (AFM) ordered to the 1D paramagnetic (PM) disordered phase ${ }^{26,27}$. In (c), the confined spinon levels $E_{i}$ shown in Fig. 4 (e) are also marked (symbols and dotted lines), and a hybridization gap of $0.68 \mathrm{meV}$ is indicated. The calculated results in (b) and (d) are given with parameters $J=3.55$ $\mathrm{meV}, \Delta=2.00$, and $g_{x}=2.79$ (see text).

pression of the spinon confinement.

In addition, the $\gamma$ mode observed at $7.72 \mathrm{meV}$ in zero field [Fig. 2(a)] shifts only slightly to higher energy and becomes broader with the increasing transverse fields. At $7 \mathrm{~T}$, the $\gamma$ mode is already suppressed.Although the origin of this mode remains unclear, it is natural to relate the $\gamma$ mode to the 3D magnetic order because it is observed only below the magnetic phase transition.

The eigenenergies of the confined acoustic and optical spinons, and of the $\gamma$ mode are summarized in Fig. प(e) as a function of the applied transverse magnetic field. Transverse-field tuning of the spinon confinement is obvious: Starting from zero field, both the acoustic and optical spinons are confined due to the inter-chain couplings and exhibit clear hierarchy of the spinon bound states. With increasing field, the inter-chain couplings are effectively reduced and the higher-level bound states are shifted downwards until the second-level collapses onto the first-level at $7 \mathrm{~T}$. The breakdown of the hierarchy, suggesting the suppression of the inter-chain couplings, is an evident signature of the field-induced phase transition $26,27$.

\section{Emergent fermions of 1D transverse-field Ising class}

The above section documents the deconfining behavior of the confined spinons in a transverse magnetic field below the phase transition at $H_{c}=7 \mathrm{~T}$. Above $H_{c}$ in the quantum disordered phase, one would expect emergent elementary excitations that characterize spin state of the disordered phase. The characteristic excitations can be revealed by performing magneto-optic experiment in high magnetic fields.

A typical absorption-coefficient spectrum is shown in Fig. [5 (a) for a transverse field of $11 \mathrm{~T}$ above the fieldinduced phase transition. One can observe three absorption maxima at $3.1,6.4$, and $8.8 \mathrm{meV}$, corresponding to the $\alpha, \beta_{-}$, and $\beta_{+}$modes, respectively. Their evolution with the transverse field is displayed in Fig. 5 (c) by a contour-plot of the absorption coefficient as a function of photon energy and transverse field, together with colored symbols highlighting their eigenenergies. The three modes $\alpha, \beta_{-}$, and $\beta_{+}$are the dominant excitations that can be unambiguously followed in higher fields.

The $\alpha$ mode, evolving from the lowest-lying confined spinon excitation $\alpha_{1}$ [Figs. B(a) and 4(e)], increases in energy with increasing fields. The $\beta_{-}$and $\beta_{+}$modes correspond to the degenerate $\beta_{1}$ mode at zero field which is split in finite fields. At $22 \mathrm{~T}$ the $\alpha$ and $\beta_{-}$modes do not cross but form a small hybridization gap of $0.68 \mathrm{meV}$ [Fig. [5(c)]. Above $22 \mathrm{~T}$, the mode $\alpha$ shifts slightly downwards while the $\beta_{-}$mode shifts upwards.

\section{DISCUSSION}

In order to understand origin of the observed modes in high fields and clarify the two branches of confined spinons, we use the standard iTEBD $\operatorname{method}^{33,34}$ to 

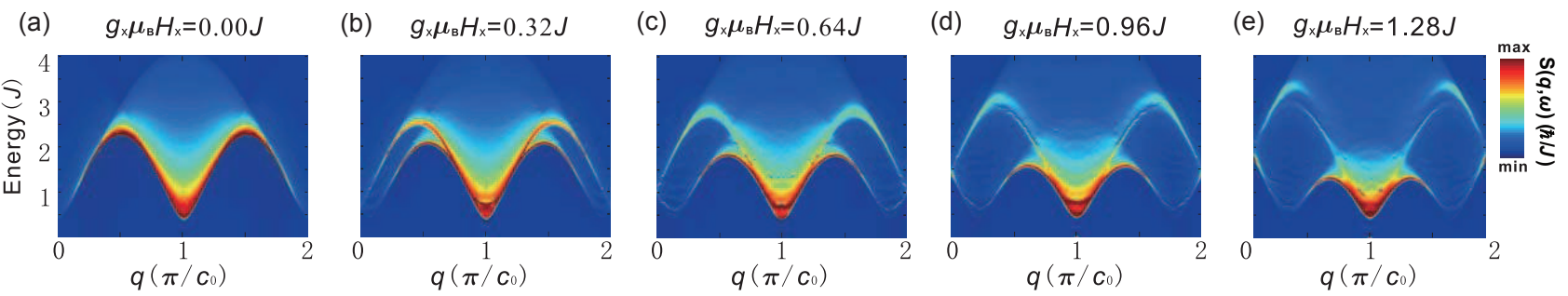

Figure 6. (a)-(e) Spin dynamic structure factor $S(q, \omega)$ [Eq. (2)] in the reciprocal space $\left(0,2 \pi / c_{0}\right)$ calculated up to $4 J$ for the 1D Heisenberg-Ising XXZ model Eq. (5) in various transverse magnetic fields $g_{x} \mu_{B} H_{x}$ of (a) 0, (b) 0.32, (c) 0.64, (d) 0.96, and (e) $1.28 J$ by the iTEBD method ${ }^{33,34}$. $c_{0}$ is the natural lattice constant of the 1D XXZ model and $J$ is the nearest-neighbor exchange interaction. The dynamic structure factor represents the dispersion relation of (a) spinons and (b)-(e) emergent fermions.

study the spin dynamics of the spin-1/2 1D HeisenbergIsing XXZ model, which describes the magnetic interactions of the cobalt spin chain. In a transverse magnetic field, the spin Hamiltonian is given by

$$
J \sum_{\langle i, j\rangle}\left(S_{x, i} S_{x, j}+S_{y, i} S_{y, j}+\Delta S_{z, i} S_{z, j}\right)-g_{x} \mu_{B} H_{x} \sum_{i} S_{x, i}
$$

where $\langle i, j\rangle$ denotes the nearest neighbors, $J$ is the nearest-neighbor intra-chain antiferromagnetic exchange interaction, and $\Delta$ refers to an Ising-like anisotropy ${ }^{37,38}$. The last term is the Zeeman interaction term with the transverse magnetic field $H_{x}$ along the $a$ axis, the Landé $g$-factor, and the Bohr magneton $\mu_{B}$.

The obtained spin dynamic structural factor (DSF) according to Eq. (21) is shown for the whole Brillouin zone at various transverse magnetic fields in Fig. 6. At zero field the known dispersion relation of spinons 10,39 is well reproduced in our calculations [Fig. 6(a)]. The dispersion relation is symmetric with respect to $q=\pi$. Two minima of the lower-energy boundary are degenerate and located at $q=0$ and $q=\pi$, respectively. In a finite transverse field, the energy at $q=0$ starts to increase and the corresponding minimum shifts to larger $q$ values, while at $q=\pi$ the energy minimum is only slightly lowered. Moreover, a clear split of the dispersion relation occurs between $q=0$ and $q=\pi$ and becomes more significant with the increasing transverse fields. These features of the dispersion relation characterize emergent fermions of the transverse-field Ising-like chain $\underline{40,41}$.

Terahertz spectroscopy is expected to probe the dispersion relation close to the $\Gamma$ point $(q=0)$ in reciprocal space, since the wavelength of terahertz electromagnetic waves is much larger than the lattice constants of $\mathrm{SrCo}_{2} \mathrm{~V}_{2} \mathrm{O}_{8}$. To compare with the experimental results, in Fig. 5(d) the obtained DSF at $q=0$ is shown as a function of energy and transverse magnetic field, which resembles the field dependence of the experimentally observed $\alpha$ mode. This indicates that the $\alpha$ fermionic excitations feature the spin dynamics at the $\Gamma$ point and the corresponding confined spinons observed at zero field belong to an acoustic branch.

To reconcile the experimental results, the DSF at $q=\pi / 2$ is also shown in Fig. 5 (d) as a function of transverse field. An excitation mode close to $8 \mathrm{meV}$ at zero field splits into two branches in the finite transverse fields, one increasing in energy while the other decreasing. Figure 5(b) shows the DSF spectra of $q=0$ and $q=\pi / 2$ which agrees very well with the absorption-coefficient spectrum of $11 \mathrm{~T}$ in Fig. 5(a). The good agreement between experiment and theory is achieved with the parameters $J=3.55 \mathrm{meV}, g_{x}=2.79$ and $\Delta=2.00$. The $g_{x}$ factor smaller than the longitudinal $g_{\|}=5.5$ and the $\Delta>1$ value reveals an Ising-like anisotropy $\underline{22}$ The exchange interaction is quite larger than the value of $J=2.8 \mathrm{meV}$ in $\mathrm{BaCo}_{2} \mathrm{~V}_{2} \mathrm{O}_{8} \stackrel{20}{\rightleftharpoons}$ while the anisotropy of the two systems is very close to each other.

These results indicate that the experimentally observed $\beta_{-}$and $\beta_{+}$modes correspond to the emergentfermion excitations at $q=\pi / 2$ of the $1 \mathrm{D}$ transverse-field XXZ model, which are the low-energy spin excitations characteristic for the quantum disordered phase. It is also clarified that the $\beta_{i}$ modes observed at zero field [Fig. 1(a)] can be assigned as confined spinons of optical branch, in analogy with the concept of optical phonons. At zero field the DSF at $q=\pi / 2$ is much larger than that at $q=0$. This explains the stronger absorption of the $\beta_{1}$ mode compared to the $\alpha_{1}$ mode, as observed in Fig. 2(a).

The observation of the $q=\pi / 2$ mode by optical spectroscopy indicates that the Brillouin zone is folded by a factor of four and translational invariance of the 1D XXZ model is broken due to the four spin-sites per unit cell in $\mathrm{SrCo}_{2} \mathrm{~V}_{2} \mathrm{O}_{8}$ [Fig. [1(g)]. The zone-folding effect also explains the experimental observation of the hybridization gap at $22 \mathrm{~T}$ [Figs. 5 (c)]. In the extended Brillouin zone the crossing of the acoustic $\alpha$ and optical $\beta_{-}$modes [Figs. [5(d)] is protected by the translational symmetry, since they are located at different $q$ vectors. Due to the broken symmetry in the material, the transverse field can lead to non-zero off-diagonal elements of the interaction matrix, and thus mixes $\alpha$ and $\beta_{-}$states and opens the hybridization gap.

With further increasing transverse fields, our calculation suggests that the lower-boundary at $q=\pi$ will be 
first gapless at $55 \mathrm{~T}$, where a transverse-field Ising quantum critical point $\underline{40.41}$ is expected to emerge. At the quantum critical point, predicted dynamical features $\underline{16.42}$ are interesting to be testified in real materials.

\section{SUMMARY}

Using high-resolution terahertz spectroscopy, we have studied low-energy spin dynamics of the Ising-like antiferromagnetic chain $\mathrm{SrCo}_{2} \mathrm{~V}_{2} \mathrm{O}_{8}$ in transverse fields up to $25 \mathrm{~T}$. According to the terahertz electrodynamic response, the transverse field-tuned spin dynamics is clarified and it can be categorized into three regimes:

i) At zero field, the spin dynamics is characterized by confined spinons. We have identified confined spinons of an optical branch in addition to an acoustic branch. Both branches can be described by a 1D Schrödinger equation with linear confining potential. Compared to the acoustic one, the optical branch is found at higher energy with larger effective mass.

ii) We have shown that the confinement of the optical and of the acoustic spinons can be tuned down by a small transverse field: The confining potential becomes shallower and the higher confinement levels are shifted closer to the lowest one. These properties can be viewed as deconfining behavior of the confined spinons, since the spinon series can be still described by the 1D Schrödinger equation and the small transverse field can be viewed as a perturbation.

iii) We have also provided experimental evidence of the suppression of the spinon confinement by large transverse field, which is manifested by the collapse of the spinon hierarchy. This, however, does not result in deconfined spinons, but leads to the emergent-fermion excitations of the 1D transverse-field Ising class, because the transverse field is too strong to be treated as perturbation and even a phase transition from the antiferromagnetic to paramagnetic phase is induced. The evolution of emergent fermions in high transverse fields has been revealed by the terahertz spectroscopy and by studying low-energy spin dynamics of the 1D Ising-like XXZ model using the method of infinite time evolving block decimation. A quantum critical point of transverse-field Ising class is predicted to occur at $55 \mathrm{~T}$ yet to be testified.

\section{ACKNOWLEDGMENTS}

We would like to thank I. Affleck, S.-W. Cheong, B. Grenier, Zhangzhen He, T. Lorenz, A. Rosch, and S. Zvyagin for stimulating discussions. We acknowledge partial support by the Deutsche Forschungsgemeinschaft via the Transregional Research Collaboration TRR 80: From Electronic Correlations to Functionality (Augsburg - Munich - Stuttgart) and the Project DE 1762/21 , and by the Chinesisch-Deutsches Zentrum für Wissenschaftsförderung. The high field experiments were supported by the HFML-RU/FOM, member of the European Magnetic Field Laboratory (EMFL). J.W., S.X., W.Y., and C.W. acknowledge the support by NSF DMR1410375 and AFOSR FA9550-14-1-0168. J.W. acknowledges the hospitality of Rice Center for Quantum Materials. C.W. acknowledges the supports from the President's Research Catalyst Awards CA-15-327861 from the University of California Office of the President, the National Natural Science Foundation of China (11328403), the CAS/SAFEA International Partnership Program for Creative Research Teams of China.

* e-mail: zhe.wang@physik.uni-augsburg.de

† e-mail: jdwu@physics.ucsd.edu

$¥$ Present address: Solid State Physics Division, Bhabha Atomic Research Centre, Mumbai 400085, India

1 T. Giamarchi, Quantum Physics in One Dimension, (Oxford Univ. Press, Oxford, 2004).

2 V. Zapf, M. Jaime, and C. D. Batista, Bose-Einstein condensation in quantum magnets, Rev. Mod. Phys. 86, 563 (2014).

3 M. Punk, D. Chowdhury, and S. Sachdev, Topological excitations and the dynamic structure factor of spin liquids on the kagome lattice, Nature Physics 10, 289-293 (2014).

4 T. Han, J. S. Helton, S. Chu, D. G. Nocera, J. A. Rodriguez-Rivera, C. Broholm, and Y. S. Lee, Fractionalized excitations in the spin-liquid state of a kagome-lattice antiferromagnet, Nature 492, 406-410 (2012).

5 B. Dalla Piazza, M. Mourigal, N. B. Christensen, G. J. Nilsen, P. Tregenna-Piggott, T. G. Perring, M. Enderle, D. F. McMorrow, D. A. Ivanov, and H. M. Rønnow, Fractional excitations in the square-lattice quantum antiferromagnet, Nature Physics 11, 62-68 (2015).

6 Z. Wang, D. L. Quintero-Castro, S. Zherlitsyn, S. Yasin, Y. Skourski, A.T.M.N. Islam, B. Lake, J. Deisenhofer, and A. Loidl, Field-induced magnonic liquid in the $3 D$ spindimerized antiferromagnet $\mathrm{Sr}_{3} \mathrm{Cr}_{2} \mathrm{O}_{8}$, Phys. Rev. Lett. 116, 147201 (2016).

7 B. Lake, D. A. Tennant, J.-S. Caux, T. Barthel, U. Schollwöck, S. E. Nagler, and C. D. Frost, Multispinon continua at zero and finite temperature in a near-ideal Heisenberg chain, Phys. Rev. Lett. 111, 137205 (2013).

8 M. Mourigal, M. Enderle, A. Klöperpieper, J.-S. Caux, A. Stunault, and H. M. Rønnow, Fractional spinon excitations in the quantum Heisenberg antiferromagnetic chain, Nature Physics 9, 435-341 (2013).

${ }^{9}$ R. Coldea, D. A. Tennant, E. M. Wheeler, E. Wawrzynska, D. Prabhakaran, M. Telling, K. Habicht, P. Smeibidl, and K. Kiefer, Quantum Criticality in an Ising Chain: Experimental Evidence for Emergent E8 Symmetry, Science 327, 177 (2010).

10 B. Lake, A. M. Tsvelik, S. Notbohm, D. A. Tennant, T. G. Perring, M. Reehuis, Ch. Sekar, G. Krabbes, and B. Büchner, Confinement of fractional quantum number par- 
ticles in a condensed-matter system, Nature Physics 6, 50 (2010).

11 J. P. Goff, D. A. Tennant, and S. E. Nagler, Exchange mixing and soliton dynamics in the quantum spin chain $\mathrm{CsCoCl}_{3}$, Phys. Rev. B 52, 15992 (1995).

${ }^{12}$ L. S. Wu, W. J. Gannon, I. A. Zaliznyak, A. M. Tsvelik, M. Brockmann, J.-S. Caux, M. S. Kim, Y. Qiu, J. R. D. Copley, G. Ehlers, A. Podlesnyak, M. C. Aronson, Orbitalexchange and fractional quantum number excitations in an f-electron metal $\mathrm{Yb}_{2} \mathrm{Pt} 2 \mathrm{~Pb}$, Science 352, 1206-1210 (2016).

13 R. Toskovic, R. van den Berg, A. Spinelli, I. S. Eliens, B. van den Toorn, B. Bryant, J.-S. Caux, and A. F. Otte, Atomic spin-chain realization of a model for quantum criticality, Nature Physics 12, 656-660 (2016).

14 R. G. Pereira, J. Sirker, J.-S. Caux, R. Hagemans, J. M. Maillet, S. R. White, and I. Affleck, Dynamical Spin Structure Factor for the Anisotropic Spin-1/2 Heisenberg Chain, Phys. Rev. Lett. 96, 257202 (2006).

15 M. Kohno, Dynamically Dominant Excitations of String Solutions in the Spin-1/2 Antiferromagnetic Heisenberg Chain in a Magnetic Field, Phys. Rev. Lett. 102, 037203 (2009).

16 J. Wu, M. Kormos, and Q. Si, Finite-temperature spin dynamics in a perturbed quantum critical Ising chain with an $E_{8}$ symmetry, Phys. Rev. Lett. 113, 247201 (2014).

17 B. Bruognolo, A. Weichselbaum, J. von Delft, and M. Garst, Dynamic structure factor of the spin- $\frac{1}{2}$ XXZ chain in a transverse field, Phys. Rev. B 94, 085136 (2016).

18 L. D. Faddeev and L. A. Takhtajan, What is the spin of a spin wave? Phys. Lett. A 85, 375-377 (1981).

19 C. M. Morris, R. Valdés Aguilar, A. Ghosh, S. M. Koohpayeh, J. Krizan, R. J. Cava, O. Tchernyshyov, T. M. McQueen, and N. P. Armitage, Hierarchy of bound states in the one-dimensional ferromagnetic Ising chain $\mathrm{CoNb}_{2} \mathrm{O}_{6}$ investigated by high-resolution time-domain terahertz spectroscopy, Phys. Rev. Lett. 112, 137403 (2014).

20 S. Kimura, H. Yashiro, K. Okunishi, M. Hagiwara, Z. He, K. Kindo, T. Taniyama, and M. Itoh, Field-induced order-disorder transition in antiferromagnetic $\mathrm{BaCo}_{2} \mathrm{~V}_{2} \mathrm{O}_{8}$ driven by a softening of spinon excitation, Phys. Rev. Lett. 99, 087602 (2007).

21 B. Grenier, S. Petit, V. Simonet, E. Canévet, L.-P. Regnault, S. Raymond, B. Canals, C. Berthier, and P. Lejay, Longitudinal and Transverse Zeeman Ladders in the IsingLike Chain Antiferromagnet $\mathrm{BaCO}_{2} \mathrm{~V}_{2} \mathrm{O}_{8}$, Phys. Rev. Lett. 114, 017201 (2015).

22 Z. Wang, M. Schmidt, A. K. Bera, A. T. M. N. Islam, B. Lake, A. Loidl, and J. Deisenhofer, Spinon confinement in the one-dimensional Ising-like antiferromagnet $\mathrm{SrCO}_{2} \mathrm{~V}_{2} \mathrm{O}_{8}$, Phys. Rev. B 91, 140404(R) (2015).

23 T. Muta, Foundations of Quantum Chromodynamics, (World Scientific Publishing, Singapore, 1987).

24 N. Ishimura and H. Shiba, Dynamical correlation functions of one-dimensional anisotropic Heisenberg model with spin 1/2. I Ising-like antiferromagnets, Prog. Theor. Phys. 63, 743 (1980).

${ }^{25}$ H. Shiba, Quantization of magnetic excitation continuum due to interchain coupling in nearly one-dimensional Isinglike antiferromagnets, Prog. Theor. Phys. 64, 466 (1980).

26 A. K. Bera, B. Lake, W.-D. Stein, and S. Zander, Magnetic correlations of the quasi-one-dimensional half-integer spinchain antiferromagnets $\mathrm{SrM}_{2} \mathrm{~V}_{2} \mathrm{O}_{8}(\mathrm{M}=\mathrm{Co}, \mathrm{Mn})$. Phys.
Rev. B 89, 094402 (2014).

27 Z. He, T. Taniyama, and M. Itoh, Antiferromagneticparamagnetic transitions in longitudinal and transverse magnetic fields in a $\mathrm{SrCo}_{2} \mathrm{~V}_{2} \mathrm{O}_{8}$ crystal. Phys. Rev. B 73, 212406 (2006).

28 E. Canévet, B. Grenier, M. Klanjšek, C. Berthier, M. Horvatić, V. Simonet, and P. Lejay, Field-induced magnetic behavior in quasi-one-dimensional Ising-like antiferromagnet $\mathrm{BaCo}_{2} \mathrm{~V}_{2} \mathrm{O}_{8}$ : A single-crystal neutron diffraction study, Phys. Rev. B 87, 054408 (2013).

29 Y. Kawasaki, J. L. Gavilano, L. Keller, J. Schefer, N. B. Christensen, A. Amato, T. Ohno, Y. Kishimoto, Z. He, Y. Ueda, and M. Itoh, Magnetic structure and spin dynam$i c s$ of the quasi-one-dimensional spin-chain antiferromagnet $\mathrm{BaCo}_{2} \mathrm{~V}_{2} \mathrm{O}_{8}$, Phys. Rev. B 83, 064421 (2011).

30 S. K. Niesen, O. Breunig, S. Salm, M. Seher, M. Valldor, P. Warzanowski, and T. Lorenz, Substitution effects on the temperature versus magnetic field phase diagrams of the quasi-one-dimensional effective Ising spin-1/2 chain system $\mathrm{BaCo}_{2} \mathrm{~V}_{2} \mathrm{O}_{8}$, Phys. Rev. B 90, 104419 (2014).

31 S. K. Niesen, G. Kolland, M. Seher, O. Breunig, M. Valldor, M. Braden, B. Grenier, and T. Lorenz, Magnetic phase diagrams, domain switching, and quantum phase transition of the quasi-one-dimensional Ising-like antiferromagnet $\mathrm{BaCo}_{2} \mathrm{~V}_{2} \mathrm{O}_{8}$ Phys. Rev. B 87, 224413 (2013).

32 S. Kimura, K. Okunishi, M. Hagiwara, K. Kindo, Z. He, T. Taniyama, M. Itoh, K. Koyama, and K. Watanabe, Collapse of magnetic order of the quasi one-dimensional Ising-like antiferromagnet $\mathrm{BaCO}_{2} \mathrm{~V}_{2} \mathrm{O}_{8}$ in transverse fields, J. Phys. Soc. Jap. 82, 033706 (2013).

${ }^{33}$ G. Vidal, Classical simulation of infinite-size quantum lattice systems in one spatial dimension, Phys. Rev. Lett. 98, 070201 (2007).

34 R. Orús and G. Vidal, Infinite time-evolving block decimation algorithm beyond unitary evolution, Phys. Rev. B 78, 155117 (2008).

35 S. R. White and I. Affleck, Spectral function for the $S=1$ Heisenberg antiferromagetic chain, Phys. Rev. B 77, 134437 (2008).

36 B. M. McCoy and T. T. Wu, Two-dimensional Ising field theory in a magnetic field: Breakup of the cut in the twopoint function, Phys. Rev. D 18, 1259 (1978).

37 J. C. Bonner and M. E. Fisher, Linear magnetic chains with anisotropic coupling, Phys. Rev. 135, A640 (1964).

38 M. E. Lines, Magnetic Properties of $\mathrm{CoCl}_{2}$ and $\mathrm{NiCl}_{2}$, Phys. Rev. 131, 546 (1963).

39 A. H. Bougourzi, M. Karbach, and G. Müller, Exact twospinon dynamic structure factor of the one-dimensional $s=1 / 2$ Heisenberg-Ising antiferromagnet, Phys. Rev. B 57, 11429 (1998).

40 D. V. Dmitriev, V. Ya. Krivnov, A. A. Ovchinnikov, and A. Langari, One-dimensional anisotropic Heisenberg model in the transverse magnetic field, J. Exp. Theor. Phys. 95, 538-549 (2002).

41 J.-S. Caux, F. H. L. Essler, and U. Löw, Dynamical structure factor of the anisotropic Heisenberg chain in a transverse field, Phys. Rev. B 68, 134431 (2003).

42 A. B. Zamolodchikov, Integrals of motion and s-matrix of the (scaled) $T=T_{c}$ Ising model with magnetic field, Int. J. Mod. Phys. A 4, 4235 (1989). 